\title{
INCREASING THE COMPETITIVENESS OF MAINTENANCE CONTRACT RATES BY USING AN ALTERNATIVE METHODOLOGY FOR THE CALCULATION OF AVERAGE VEHICLE MAINTENANCE COSTS
}

\author{
STEPHEN CARSTENS \\ stephcar@global.co.za \\ RCBM (Pty) Ltd
}

\begin{abstract}
Companies tend to outsource transport to fleet management companies to increase efficiencies if transport is a non-core activity. The provision of fleet management services on contract introduces a certain amount of financial risk to the fleet management company, specifically fixed rate maintenance contracts. The quoted rate needs to be sufficient and also competitive in the market. Currently the quoted maintenance rates are based on the maintenance specifications of the manufacturer and the risk management approach of the fleet management company. This is usually reflected in a contingency that is included in the quoted maintenance rate.
\end{abstract}

An alternative methodology for calculating the average maintenance cost for a vehicle fleet is proposed based on the actual maintenance expenditures of the vehicles and accepted statistical techniques. The proposed methodology results in accurate estimates (and associated confidence limits) of the true average maintenance cost and can be used as a basis for the maintenance quote.

\section{INTRODUCTION}

\section{Background}

Recent inflationary pressures on South Africa's economy have resulted in a number of prime interest rate increases, slowing down the economic growth rate (Isa, 2007: 2). In this economic environment South African companies have become increasingly aware of the associated operating cost increases, and special attention is given to the cost of non-core activities such as transport (Windell, 2007). Transport costs may result from freight and/or company cars and could add a significant proportion to total company costs. For example, from a freight transport perspective the cost may constitute $63.1 \%$ of the total logistics cost of a company (Botes, Jacobs \& Pienaar, 2006: 16).

One way to address the cost of transport is through efficient fleet management (Oliver, 2007: 4). Fleet management can be defined as "the long term reduction of a customer's total material handling costs, which results in increased efficiency via a planned program that optimises the economic life of the equipment" (Bartlett, 2007: 33). 
Efficient fleet management can be achieved through the application of specialised resources such as fleet management systems which in most instances require significant capital investments. However, a company may outsource the fleet management function to third party fleet management companies on contract to acquire the benefits of these resources, but avoid the capital investment (Ryan, 2006). The rate of these fleet management services could either be fixed for the contract period or vary according to a predetermined measure.

The provision of fleet management services on a fixed rate contract introduces a certain amount of financial risk to the service provider, i.e. the fleet management company (Standard Bank, 2007). Although fleet management companies provide various fleet management functions on contract, maintenance cost is one of the vehicle costs that is difficult to manage efficiently, because of the uncertainty involved in ad hoc maintenance. In these types of contracts the fleet management company is required to quote a fixed rate (cost) for the service over the contract period before the contract takes effect.

\section{Fixed rate maintenance contracts}

The quoted rate for a fixed rate maintenance contract should be sufficient to cover all scheduled and unscheduled maintenance and repairs expenditure of the vehicle over the contract period (Standard Bank, 2007). In addition, the rate needs to take into account probable increases in the prices of spares, parts and labour. Simultaneously, the quoted rate should be competitive in the fleet management market.

The current methods of calculating the maintenance rate depend mainly on the maintenance specifications of the manufacturer (Smith, 2007) and the risk management principles followed by the fleet management company (Braun, 2005). This could lead to an overprovision for maintenance and a non-competitive maintenance rate. An empirical study indicated that these methods could lead to a 30\% variance in quoted maintenance rates (Carstens, 2000: 115).

Furthermore, the majority of these methods do not allow the fleet management company to attach any measure of statistical confidence to the calculated rate. Therefore, the fleet management company would not be able to adjust the calculated rate with known confidence in a competitive environment.

The number of vehicles managed by a fleet management company and the length of the contracts (distance and time) render the efficient management of these contracts critical. The financial impact of an incorrect maintenance rate can be illustrated as follows: A $1 \mathrm{cent} / \mathrm{km}$ underprovision for maintenance of a fleet of 1000 vehicles over 120000 km/36-month fixed rate maintenance contracts amounts to a total underprovision of R1.2 million over the contract period. 


\section{RESEARCH STRATEGY}

\section{Proposed methodology for the calculation of average maintenance costs}

An alternative methodology for calculating an average maintenance cost is proposed that uses actual maintenance costs per vehicle type (as recorded by the fleet management company) to estimate average maintenance cost figures at selected kilometre intervals based on the relationship between a vehicle's maintenance cost and the associated distance travelled.

The proposed methodology is based on accepted statistical techniques, i.e. simple linear regression and time series analysis. Regression analysis is relatively easy to use and most existing spreadsheet programs have regression analysis available as a function. These software packages provide the user with the main regression analysis outputs that are necessary to describe the relationship between maintenance cost and the distance travelled that can be used to estimate average maintenance costs at any kilometre distance.

Figure 1 shows typical maintenance costs for a specific vehicle type (cumulative costs $v$ kilometre).

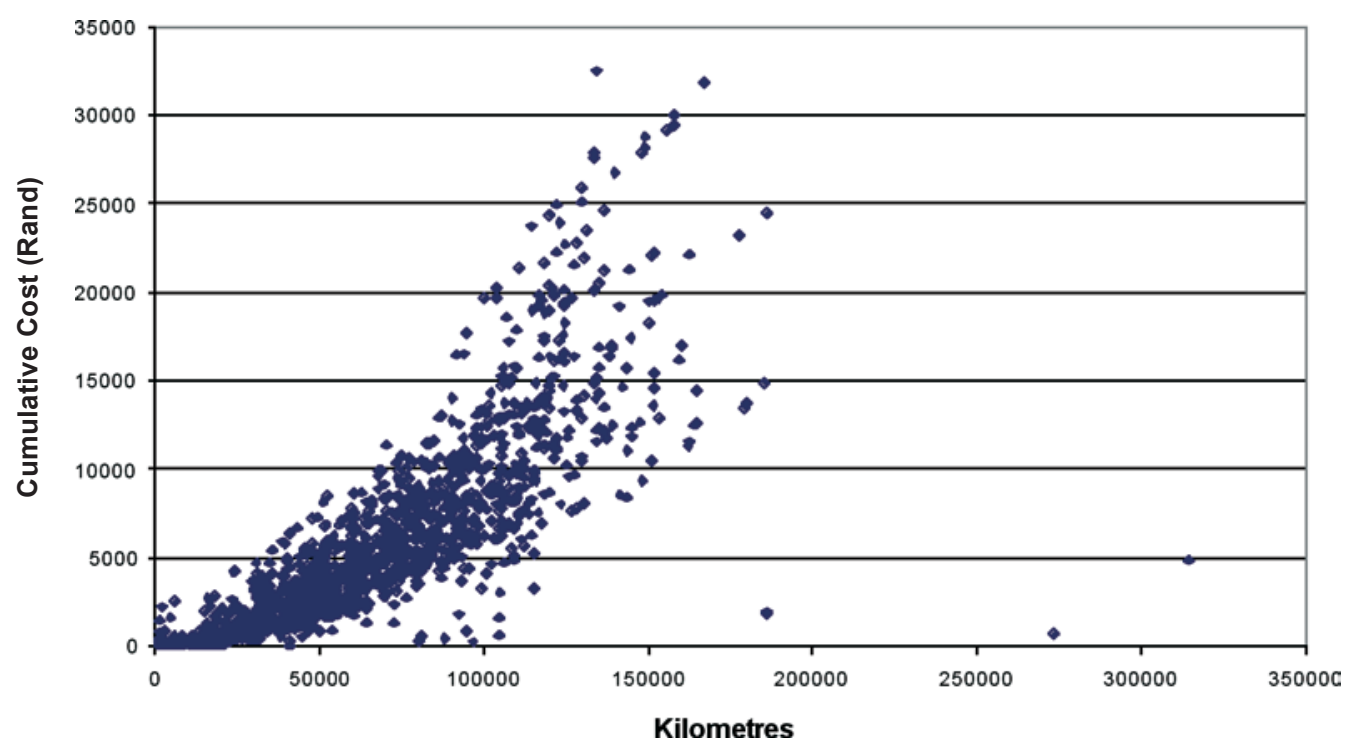

Figure 1: Maintenance costs for a specific vehicle type

The information in Figure 1 was supplied by a fleet management company and consists of 1644 maintenance expenditure records of 193 identical vehicles on maintenance contracts over a period of 4 years. The data set includes vehicles at various stages in the maintenance contracts (Carstens 2000: 154). Cumulative maintenance costs were used to establish a maintenance cost trend in terms of distance travelled over the vehicle life.

Fleet management companies capture the maintenance costs on the date of the expenditure. Therefore, it would be incorrect to simply add the individual maintenance costs to obtain cumulative maintenance costs since the effect of inflation in parts and labour is not taken 
into account (Carstens, 2000: 180). The maintenance costs from different years should be inflated to reflect present values. Although various methods can be used to inflate the maintenance costs, the data used in this study was inflated according to a custom-designed price index based on the service costs including parts and labour of the specific vehicle model (Carstens, 2000: 182).

Outliers (extreme data values) could be expected in the data. These values could result from capturing errors or out-of-the-ordinary maintenance expenditure (Carstens, 2000: 192). The proposed methodology succeeds in detecting all the relevant outliers. However, these values should be evaluated to decide whether they can be omitted from the regression - the removal of the outliers generally increases the accuracy of the fitted model (Hogg \& Ledolter, 1992: 377).

Linear regression analysis is based on the assumption of a linear relationship between the variables (dependent and independent variable). However, Figure 1 does not show a highly linear relationship between cumulative maintenance cost (dependent variable) and distance travelled (independent variable). In many cases, the transformation of the dependent and/or independent variables may lead to the simplification of the relationship between the variables. One such transformation of variables that is often used is the logarithmic transformation (Gujarati, 2003: 176). The logarithmic transformation tends to increase the linearity between the variables and decrease the spread in the observations (variance) (Gujarati, 2003: 421).

If the independent variable is indicated by $x$ and the dependent variable by $y$, the term linear regression implies that the mean of $y$ is linearly related to $x$ in the following form (Walpole \& Myers, 1978: 282):

$$
y=\alpha+\beta x
$$

The ordinary least squares estimates $a$ and $b$ lead to the estimated (fitted) regression line, i.e. (Walpole et al. 1978: 282)

$$
\hat{y}=a+b x+e
$$

where $\hat{y}$ indicates the estimated value of the dependent variable and e indicates the residual (error term). The residuals are differences between the observations $y$ of the dependent variable and the fitted values $\hat{y}$.

Simple linear regression analysis is based on a number of assumptions, i.e. that the expected value of the residuals is zero (Gujarati, 2003: 67), a constant variance of the residuals 
(homoscedastic) (Gujarati, 2003: 68), and that the residual values are not correlated, i.e. zero autocorrelation (Gujarati, 2003: 70). These assumptions can be verified through residual analysis which includes residual plots and time series analyses (Hogg et al. 1992: 364).

In order to evaluate how well the estimated regression line fits the data, various goodnessof-fit measures can be calculated. One such measure is the coefficient of determination, $\mathrm{R}^{2}$ that expresses the amount of variation in the dependent variable explained by the linear regression model (Wilson \& Keating, 2002: 161). The value of $\mathrm{R}^{2}$ ranges from 0 to 1 where a value of 1 means that all the variation in the independent variable is explained by the linear regression model (deterministic model). An $\mathrm{R}^{2}$ value of 0 indicates that the linear regression model explains none of the variation in the dependent variable (Wilson et al. 2002: 162).

Another aspect of the evaluation of the regression model is whether the parameters are significantly different from zero (Wilson et al. 2002: 158). Hypothesis tests are widely used to establish whether the estimated regression parameters, a and b, are significantly different from zero (Wilson et al. 2002: 160).

The presence of outliers could affect the parameter estimates severely in linear regression analysis, and it is therefore important to detect outliers in a data set. "A single observation that lies very far to one side of the other values is called an outlier" (Hogg et al. 1992: 19). It is also important to establish the cause of the outliers. Outliers could result from data errors, i.e. data capturing errors or from the data itself, for example, the replacement of a gearbox of a vehicle very early in the life of the vehicle. The cause of the outlier determines whether such an outlier should be excluded from the analysis or not (Hogg et al. 1992: 377).

Although various methods of detecting outliers exist, a method of outlier detection proposed by Belsley, Kuh and Welsch (1980: 20) could be applied to the data. They indicated that the studentised residuals have an approximate t-distribution (or a normal distribution if $\mathrm{n}$ is moderately large) which means that the significance of any single studentised residual can be assessed.

The application of this approach is possible since the available information of the fleet management company normally consists of a large number of observations that allows the use of the properties of the standard normal distribution as is required by this method. This process can be applied in a recursive manner until an optimum estimated regression line is obtained (Carstens, 2000: 204). 


\section{DISCUSSION}

\section{Results of the application of the proposed methodology for the calculation of average maintenance costs based on research by Carstens (2000)}

Linear regression analysis is based on the assumption of a linear relationship between the variables (dependent and independent variable). However, Figure 1 does not show a highly linear relationship between cumulative maintenance cost (dependent variable) and distance travelled (independent variable). A correlation coefficient of 0.68 was calculated for the cumulative maintenance costs and their associated kilometre values in this example.

Figure 2 shows the transformed data (logarithmic transformation).

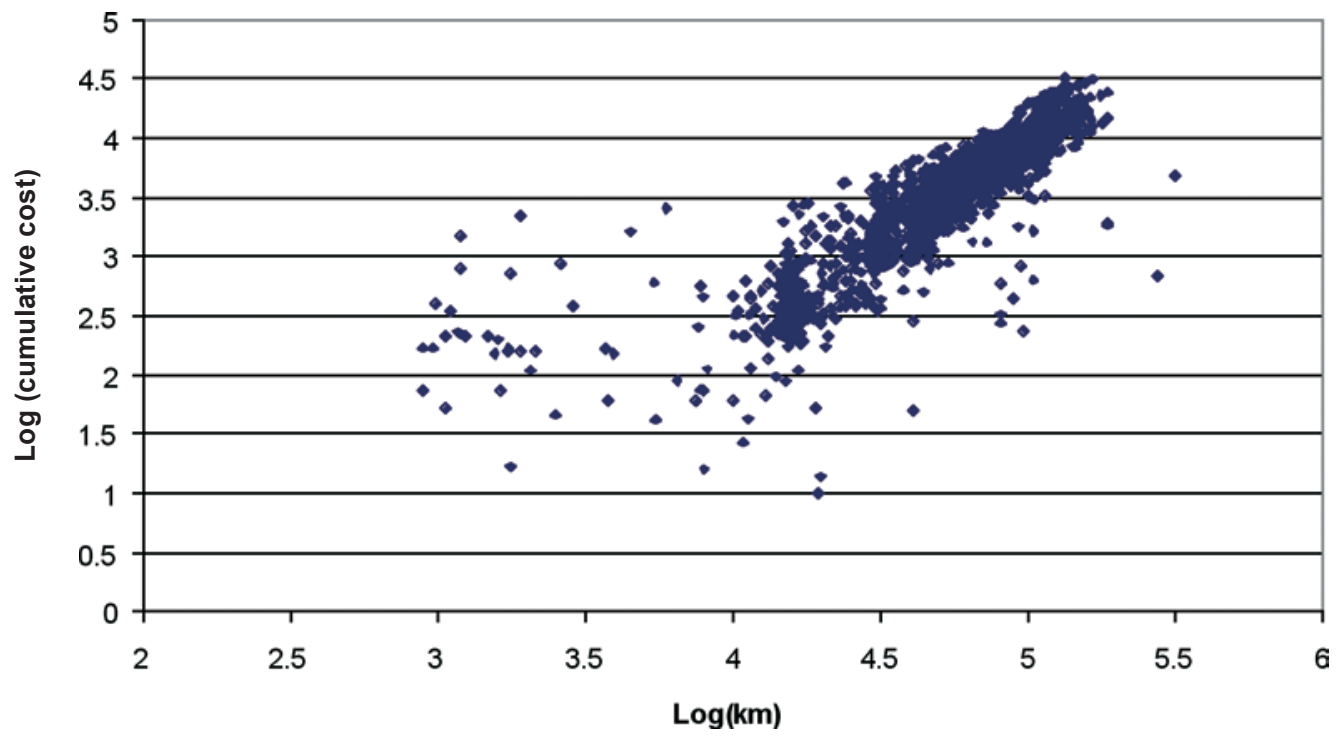

Figure 2: Logarithmic transformation of data

The associated correlation coefficient is 0.88 which indicates a relatively strong linear relationship between the variables. It is also evident that the transformation of the variables resulted in a decrease in the variance of the dependent variable which also satisfies one of the assumptions of simple linear regression.

Regression analysis of the transformed data resulted in the following estimated regression equation:

$$
\log (\text { cost })=0.15498+1.12407 \log (\mathrm{km})
$$

An $\mathrm{R}^{2}$ value of 0.77 was obtained indicating that $77 \%$ of the variation in the dependent variable (log[cost]) is explained by the linear regression line. However, various factors related to the regression assumptions could affect the $\mathrm{R}^{2}$ value negatively.

Residual analyses (residual plots and time series analyses) indicated that relatively high levels of autocorrelation were present implying that the regression assumptions were not 
satisfied. The initial analysis of the data indicated the presence of outliers which could negatively affect the parameter estimates.

The method proposed by Belsley et al. (1980) was applied to the data set and 73 observations were found to be outliers. An analysis of the outliers indicated that a number of the outliers were related to unexpected maintenance cost figures, for example a cumulative cost of only R15 at approximately $30000 \mathrm{~km}$. Other outliers resulted from data capturing errors, for example one maintenance expenditure was recorded at $34753 \mathrm{~km}$ and the next maintenance expenditure was recorded at $309431 \mathrm{~km}$. The method was applied recursively and the observations related to the outliers were subsequently removed from the data set.

Simple linear regression analysis on the reduced data set resulted in the following regression equation:

$$
\log (\cos t)=-1.10208+1.40358 \log (\mathrm{km})
$$

The associated $\mathrm{R}^{2}$ value was 0.97129 and the residual analyses indicated that the removal of all the outliers reduced the presence of autocorrelation to such an extent that it can be ignored. The residual analyses results were as follows:

Durbin-Watson statistic 1.84373

Calculated autocorrelations (first 10 lags):

$\begin{array}{lll}\text { Lag } & 1 & 0.07729 \\ & 2 & 0.04063 \\ 3 & -0.00639 \\ 4 & 0.07475 \\ 5 & 0.03896 \\ 6 & 0.07201 \\ 7 & 0.05593 \\ 8 & -0.01818 \\ 9 & -0.1250 \\ 10 & 0.03053\end{array}$

The tests of significance of the regression analysis parameters indicated that the regression line has a high quality, i.e. $a$ and $b$ are good estimates of $\alpha$ and $\beta$. The results of the hypothesis tests were as follows:

\section{Hypothesis test for a}

HO: $\alpha=0$

$\mathrm{H} 1: \alpha \neq 0$

$t_{0.005}=2.576 \quad(n-2=1102$ degrees of freedom $)$

calculated t-value $=13.88$ 


\section{Hypothesis test for $b$}

$$
\begin{aligned}
& H 0: \beta=0 \\
& H 1: \beta \neq 0 \\
& t_{0.005}=2.576(n-2=1102 \text { degrees of freedom }) \\
& \text { calculated } t \text {-value }=113.40
\end{aligned}
$$

The proposed methodology allows for the calculation of confidence intervals at selected confidence limits for the parameters and the estimated average maintenance cost. Figure 3 shows the estimated average maintenance costs as well as its associated $95 \%$ confidence intervals.

Figure 3 shows the estimated average maintenance costs (cents/km) and confidence intervals.

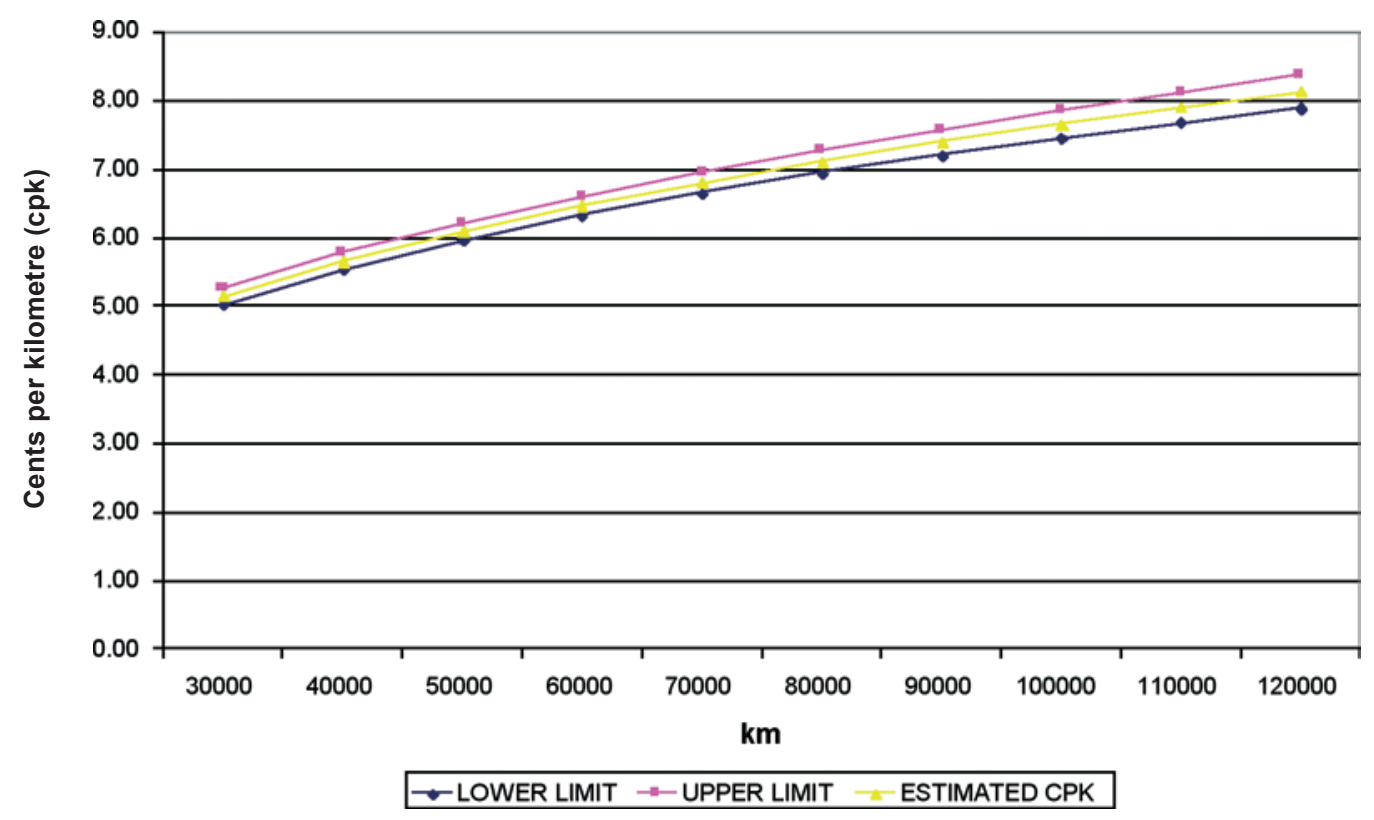

Figure 3: Estimated average maintenance costs and confidence levels

\section{Model evaluation based on research by Carstens (2000)}

Although the proposed methodology resulted in statistically sound estimates it was important to compare the estimated average maintenance cost to a calculated average maintenance cost based on the actual data in terms of cents $/ \mathrm{km}$.

To this end a subset of the data (after removal of the outliers) was randomly selected for the evaluation process and the rest of the data was used for regression analysis. The subset contained information that related to $20 \%$ of original data or 25 vehicles.

Regression analysis on $80 \%$ of the original data resulted in the following regression equation $\left(R^{2}=0.92107\right)$

$$
\log (\cos \mathrm{t})=-0.76685+1.331023 \log (\mathrm{km})
$$


The estimated regression line can be used to estimate the average cumulative maintenance cost at selected kilometre distances. For example, if a kilometre value of 30000 is selected, the cumulative maintenance cost estimate is as follows:

$$
\begin{aligned}
\log (\text { cost }) & =-0.76685+1.331023 \log (\mathrm{km}) \\
& =-0.76685+1.331023 \log (30000) \\
& =5.1888
\end{aligned}
$$

By taking the inverse logarithms the estimated cumulative maintenance cost is equal to R1 544.37 or 5.15 cents $/ \mathrm{km}$.

Table 1 shows the result of the estimated average maintenance cost for the fleet (80\% of the data), as well as the calculated average maintenance cost for the fleet comprising $20 \%$ of the data.

Table 1: Model evaluation - separate data sets (Carstens, 2000: 239)

\begin{tabular}{|l|c|c|}
\hline & Log(cost) & $\begin{array}{c}\text { Maintenance cost } \\
\text { (cents/km) }\end{array}$ \\
\hline Estimated average & 5.4770 & 4.3425 \\
\hline Calculated average & 5.4099 & 4.6334 \\
\hline
\end{tabular}

The same approach was followed with a different subset of the data and Table 2 shows the results of this comparison.

Table 2: Model evaluation - second subset used for evaluation (Carstens, 2000: 241)

\begin{tabular}{|l|c|c|}
\hline & Log(cost) & $\begin{array}{c}\text { Maintenance cost } \\
\text { (cents/km) }\end{array}$ \\
\hline Estimated average & 5.4458 & 4.1026 \\
\hline Calculated average & 5.4736 & 4.3743 \\
\hline
\end{tabular}

The maintenance cost evaluation indicates that the proposed methodology resulted in a 0.28 cents $/ \mathrm{km}(6.3 \%)$ underestimation on average of the 'true' average maintenance cost for the vehicle fleet. 


\section{CONCLUSION}

In the current South African economy companies are focusing on outsourcing non-core activities such as transport which may contribute a significant proportion to total cost. Proper fleet management can be used as a tool to manage these costs and numerous fleet management companies provide these services, of which maintenance management is one, on contract.

The provision of this service on a fixed rate introduces a certain element of financial risk to the fleet management company as a result of ad hoc maintenance expenditure. Fleet management companies endeavour to negate this risk by using the vehicle manufacturer's maintenance specifications for service and the inclusion of a contingency as basis for the maintenance rates.

The proposed methodology for the calculation of average maintenance costs is based on well-known statistical techniques and the fleet management company's actual maintenance data, i.e. costs and distances (Carstens, 2000: 262).

The proposed average maintenance cost estimation methodology provides accurate estimates of the actual average maintenance cost per vehicle type that can be used as basis for the quoted maintenance rate by including inflationary measures to the estimated average maintenance cost over the contract period.

The proposed methodology for estimating the average maintenance cost of a vehicle could be applied with little extra time and expenses and would result in a more competitive maintenance rate. The benefits of using this methodology are the following:

- The proposed methodology could be used by fleet management companies to calculate average maintenance costs for passenger and commercial vehicle fleets.

- Companies with their own vehicle fleets could use the proposed methodology to calculate benchmarks for fleet maintenance costs. The methodology could be applied to any fleet size. However, the success of the methodology depends on the number of observations. Therefore, at least 100 observations should be included due to the variance inherent in maintenance cost data (Carstens, 2000: 276).

- The proposed methodology could be used to detect extreme data values.

- The calculated confidence limits would enable a fleet management company to manage the risk efficiently.

- The quoted maintenance rate could be adjusted with confidence by utilising the calculated confidence limits. 


\section{REFERENCES}

Bartlett, G. 2007. Key to the future. FOCUS on transport and logistics, November 2007: 33-36.

Belsley, D.A., Kuh, E. \& Welsch, R.E. 1980. Regression Diagnostics: Identifying Influential Data and Sources of Collinearity. New York: John Wiley \& Sons.

Botes, F.J., Jacobs, C.G. \& Pienaar, W.J. 2006. Macro-economic perspective. The third Annual State of Logistics Survey for South Africa. CSIR.

Braun, M. 2005. Paying fortrucks. Fleetwatch. [Online]. Available from: http://www.fleetwatch. co.za/magazines/Feb2005/32-Paying\%20for\%Trucks.html (Accessed 30 November 2007).

Carstens, S.C. 2000. A transport economic appraisal of a methodology to calculate maintenance contract rates for vehicle fleets. Unpublished doctoral thesis. Johannesburg: Rand Afrikaans University.

Gujarati, D.N. 2003. Basic Econometrics. New York: McGraw-Hill.

Hogg, R.V. \& Ledolter, J. 1992. Applied statistics for engineers and scientists. New York: Macmillan.

Isa, M. 2007. Rate hikes 'taking a toll on growth'. Business Day, 27 November 2007: 2.

Oliver, V. 2007. Fleet Management. FOCUS on transport and logistics, April 2007: 4.

Ryan, C. 2006. Road to savings. Financial Mail. [Online]. Available from: http://www.fm.co. za/cgi-bin/pp-print.pl (Accessed 29 November 2007).

Smith, E. 2007. [Discussion on maintenance rate calculation]. (Personal communication, 4 December 2007).

Standard Bank. 2007. Full Maintenance Leasing. [Online]. Available from: http://www. standardbank.co.za/SBIC/Frontdoor_02_01/0,2345,7375713_12646267_0,00.html (Accessed 29 November 2007).

Walpole, R.E. \& Myers, R.H. 1978. Probability and Statistics for Engineers and Scientists. New York: Macmillan.

Wilson, J.H. \& Keating, B. 2002. Business Forecasting. New York: Macmillan.

Windell, C. 2007. Strategic wheels. Financial Mail. [Online]. Available from: http://www. fm.co.za/cgi-bin/pp-print.pl (Accessed 29 November 2007). 